\title{
Effects of high altitude: physiological adaptations of the heart and lungs
}

\begin{abstract}
Abbreviations: HIF, hypoxia-inducible factor; VEGF, vascular endothelial growth factor; EPO, erythropoietin; AMS, acute mountain sickness; HAPE, high-altitude pulmonary edema; HACE, high-altitude cerebral edema
\end{abstract}

\section{Editorial}

Mountains are defined as landforms higher than 600 meters. As a consequence of the increased altitude, the barometric pressure falls and the environmental partial pressure of inspired oxygen decreases, with consequent ambient hypoxia. This, in combination of low temperature, low humidity, increased solar radiations and presence of wind, in association with strong physical activities, imposes the human body important physiological adaptations affecting primarily the cardiovascular and respiratory systems. Physical modifications begin to be significant over 2500 meters. In normal subjects, the variability of this response may be very high and generally it is well tolerated. On the contrary, these adjustments may induce major problems in patients with preexisting cardiovascular diseases in which the functional reserves are already limited.

This article focus on the acute effects of altitude exposure as occurs in tourists, trekkers and mountaineers who normally reside at low level (lowlanders). The initial response to reduced partial pressure of oxygen is the increase in depth and rate of breathing, which results in an increase in alveolar ventilation. This is brought about by hypoxic stimulation of the peripheral chemoreceptors, mainly the carotid bodies, which sense the low $\mathrm{PaO}_{2}$ in the arterial blood. Hyperventilation reduces the alveolar $\mathrm{PCO}_{2}$ (hypocapnia) with consequent respiratory alkalosis. The kidneys correct the alkalinity of the blood over a few days by removing alkali (in the form of bicarbonate ions, $\mathrm{HCO}_{3}-$ ) from the blood. As ventilation increases, $\mathrm{PaCO}_{2}$ drops, $\mathrm{pH}$ increases and the central receptors activity subsides. Nocturnal Cheyne-Stokes breathing is a common experience when sleeping at high altitude. It results from the fluctuations of $\mathrm{PaO}_{2}$ and $\mathrm{PaCO}_{2}$ that are exaggerated during sleep, causing alternating periods of apnea and hyperventilation.

The first cardiovascular response to hypoxia is an increase in heart rate and in cardiac output with no changes in stroke volume, and the arterial blood pressure may temporarily increase. After a few days of acclimatization, cardiac output reduces to normal values, with still increased heart rate, so that stroke volume is decreased. In the same time the systemic vascular resistances increase as a response of the adrenal medullary activity and the systemic arterial pressure increase, too. As a consequence of these adaptations, myocardial workload and oxygen demand increase. Because the coronary oxygen extraction is normally physiologically high already at low altitude, the myocardium to adapt to this increased request may almost exclusively act on coronary vasodilatation enhancing coronary blood flow. Ultimately, global systolic indices of ventricular function are preserved or only slightly depressed, with altered diastolic filling pattern. Even if the relationship between workload, cardiac output and oxygen uptake is preserved, a decrease in maximal oxygen consumption and in maximal cardiac output are observed, which is minimal in acute hypoxia but is more important after acclimatization. ${ }^{1-4}$ Despite all
Volume I Issue 6 - 2014

\author{
Enrico Donegani \\ UIAA MedCom (Medical Commission of the Union \\ International des Associations d'Alpinisme-International \\ Mountaineering and Climbing Federation), Switzerland
}

Correspondence: Enrico Donegani, UIAA MedCom (Medical Commission of the Union International des Associations d'Alpinisme-International Mountaineering and Climbing Federation), Switzerland, Email donegani@hotmail.com

Received: December 08, 2014 | Published: December 14, 2014

these consequences, these adaptations are well tolerated and the high altitude exposure doesn't carry risks of myocardial ischemia in healthy subjects. ${ }^{1}$

But there are also intracellular changes that operate to reduce injuries of hypoxia and provide sufficient oxygenation when a subject is exposed to altitude. In hypoxic environment, humans are able to switch on activation of numerous genes to increase oxygen delivery. Recently it has been shown that the hypoxia-inducible factor (HIF), a family of transcription factors, plays a pivotal and fundamental regulatory role in these homeostatic changes both at systemic and cellular levels. HIF acts as the transcriptional activator of erythropoietin (EPO), which increases red blood cell production, vascular endothelial growth factor (VEGF), which stimulates vascular development, and other genes which increase glucose transport and glycolysis to produce energy in the absence of oxidative phosphorylation. $^{5}$

Another consequence of high altitude is pulmonary hypertension. The increase pressure in pulmonary artery is caused by the hypoxic vasoconstriction of pulmonary small arteries and veins and this response is very variable among humans. The degree of pulmonary hypertension is generally mild and does not contribute to the symptoms of acute mountain sickness (AMS). It can occur in tourists, as well as in hikers, skiers, and mountaineers. Interestingly, the increase in pulmonary artery pressure occurs both in individuals with AMS and in those who remain asymptomatic after the climb. Excessive pulmonary vasoconstriction play a role in the development of early high-altitude pulmonary edema (HAPE) and late within weeks right heart failure at high altitude. ${ }^{1-3,5}$

Lowlanders who ascend to medium or high altitudes may develop some degree of AMS, and the common symptoms are headache, sleep disorders, gastrointestinal disorders and dizziness. The degree of susceptibility to this illness varies and in those with vigorous response it may lead to two potential lethal ones, HAPE and high-altitude cerebral edema (HACE).The main cause is hypoxemia and, thus, the treatment is oxygen administration and, in severe cases, in addition to appropriate pharmacological treatment if available, a return to lower altitudes. Acetazolamide administration is the most widely accepted prophylaxis. Staging the ascent attenuates the symptoms of AMS and, 
thus, is recommended as a way to prevent the most serious clinical conditions as HAPE and HACE. ${ }^{1}$

\section{Acknowledgments}

None.

\section{Conflicts of interest}

Authors declare that there is no conflict of interest.

\section{References}

1. Naeije R. Physiological adaptation of the cardiovascular system to high altitude. Prog Cardiovasc Di. 2010;52(6):456-466.
2. Bartsch P, Gibbs JS. Effect of altitude on the heart and the lungs. Circulation. 2007;116(19):2191-2202.

3. Smith TG, Robbins PA, Ratcliff PJ. The human side of hypoxia inducible factor. Br J Haematol. 2008;141(3):325-334.

4. Scherrer U, Allemann Y, Jayet PY, et al. High altitude, a natural research laboratory for the study of cardiovascular physiology and pathophysiology. Prog Cardiovasc Dis. 2010;52(6):451-455.

5. Donegani E, Hillebrandt D, Windsor J, et al. Pre existing cardiovascular conditions and high altitude travel. Consensus statement of the UIAA Medical Commission. Trav Med Infec Dis. 2014;12(3):237-252. 